\title{
Ultrastructure of the Enamel Organ of the Upper Major Incisor of the Rabbit
}

\author{
By \\ Shigenori OKADA \\ Department of Anatomy, Osaka Dental University, \\ 1-47 Kyobashi, Higashi-ku, Osaka 540, Japan \\ (Director: Prof. Y. Ohta) \\ -Received for Publication, June 15, 1983-
}

Key words: Enamel organ, Ultrastructure, Incisor, Rabbits.

Summary: The present paper deals with the ultrastructure of the amelogenesis of the upper major incisor of the rabbit. Ultrastructural changes in the enamel organ of the rabbit incisor, compared to those of the rat incisor, are described on the basis of a classification into six zones/ stages, ranging from the growing end to the junctional epithelium, as follows: proliferation, differentiation, secretion, early maturation, late maturation and regression.

The inner enamel epithelial cells of the proliferation stage had wide intercellular spaces with the mitochondria located distally towards the dental papillary cell. The metabolic pathway was therefore considered to be established through this distal site. During the differentiation and secretion stages, the nucleus and mitochondria were located on the basal side (towards the stratum intermedium), but the r-ER and Golgi apparatus were on the distal side. The intercellular spaces became narrower and terminal webs were found on both the basal and distal sides. Numerous secretory granules were formed in the well-developed Golgi apparatus. The Tomes' process disappeared in the early maturation stage, and the enamel matrix began to be absorbed. In the late maturation stage, respective groups of ruffle-ended and smooth-ended ameloblasts were found one after the other three or four times. Intracellularly, a network arrangement of tonofibrils was observed in the regression stage. The cells of the stratum intermedium in both the differentiation and secretion stages prolonged their cellular processes, being interconnected by many desmosomes, and surrounded the basal cone-like projection of the ameloblasts. In the early and late maturation stages, flat-arranged papillary cells in contrast to those of the rat, contacted the blood capillaries. In the regression stage, the tonofibrils ran along the cell axis and the half desmosomes paralleled the basement membrane facing the surrounding connective tissue. The stellate reticulum began to decrease suddenly in the differentiation stage and finally disappeared in the middle of the secretion stage. The outer enamel epithelial cells were transformed into a cylindrical, cubical and flattened shape in the proliferation stage, and arranged in a wavy course, holding fenestrated blood capillaries in the differentiation and secretion stages.

The enamel formation of the rabbit incisor ended within a short period, in accordance with a shorter, early maturation and longer, late maturation, as well as other characteristic features of the enamel organ.

The incisors of the rat and rabbit are permanently growing teeth. It is thus possible to observe all the developmental stages of enamel formation at one time by using this tooth.

The rabbit was formerly assigned to the
Rodentia (Simplicidentata), but has more recently been distinguished under a separate order, the Lagomorpha (Duplicidentata), based on the characteristics of the dental formula, mastication and genital system. Some similarities between rabbits and rats 
may be present in the histological structures of their teeth and periodontal tissues. The amelogenesis of some rodents including the rabbit was investigated under the light microscope by Suga (1959). The present study attempts to elucidate the continuous ultrastructural changes of the enamel organ cells of the rabbit major incisor and to compare the results with previous findings for the rat.

\section{Materials and Methods}

Twenty-five adult rabbits were used in this study. The rabbits were perfused from the common carotid arteries with $2.5 \%$ glutaraldehyde in $0.1 \mathrm{M}$ phosphate buffer for 2 hours. The incisors and surrounding tissues were carefully dissected out and decalcified in 5\% EDTA containing $0.2 \mathrm{M}$ sucrose at $4^{\circ} \mathrm{C}$ for one week. Materials from 20 of the subjects were postfixed with $1 \%$ osmic acid for preparation of electron microscopic samples. These were ultrathin-sectioned after embedding in SPURR resin, stained with uranyl acetate and lead citrate, and examined by TEM under a JEOL-100S. The materials from the remaining five subjects were used for preparing serial sections of $15 \mu \mathrm{m}$ in thickness after celloidin embedding.

\section{Findings}

In relation to the developmental process of the amelogenesis of the rat incisor described by other workers, as well as the developmental changes of the component cells of the enamel organ, the present descriptions are given on the basis of a classification into the following six zones/stages: proliferation, differentiation, secretion, early maturation, late maturation and regression (Figs. 1, 2).

\section{Proliferation zone/stage}

This zone was located in the growing end of the enamel organ, where the enamel organ cells were clearly divided into four layers: the inner enamel epithelium, the stratum intermedium, the stellate reticulum, and the outer enamel epithelium (Figs. 3, 4). Here, a continuous basement membrane separated the enamel organ from the pulp as well as the surrounding connective tissue.

The inner enamel epithelial cells (Fig. 2-I) appeared to be shortly cylindrical (15 20 $\mu \mathrm{m}$ in height), with numerous intercellular processes, between which no kinds of attachments were observed (Fig. 3). The nucleus was elongated oval and located in the center of the cytoplasm (Fig. 3). A few mitochondria were located distally towards the dental papillary cells, the Golgi apparatus around the nucleus, and many free ribosomes and a little rough endoplasmic reticulum (r-ER) were also seen (Figs. 5, 6). The distal end of each inner enamel epithelial cell was explanded in a table-like shape and blocked the intercellular spaces diaphragmatically (Fig. 3). The distal basement membrane revealed a wavy course with half desmosome-like structures (Fig. 6). From this membrane, the microfilaments (nonstriated border of Reith 1967) gradually increased in length $(100 \mu \mathrm{m})$, being parallel and dense, and ran towards the dental papillary cells at right angles (Fig. 6).

The cells of the stratum intermedium, being cuboid, were observed as two or three layers, and expanded their cellular processes into wide intercellular spaces, with a few desmosomes between adjacent processes (Fig. 3). The cytoplasmic organelles of these cells as well as cells of the stellate reticulum were not yet highly developed.

The outer enamel epithelial cells were observed as one or two layers, more flattened as their development advanced (Fig. 4). Between adjacent processes, a few 
desmosomes were seen and microvilli projected into the intercellular spaces (Fig. 4). The basement membrane was homogeneously smooth in appearance, differing from that of the inner enamel epithelial cell, but no half desmosome-like structures were seen. The cytoplasm contained many free ribosomes, a few mitochondria, as well as tiny amounts of ER and Golgi apparatus around the nucleus (Fig. 4).

\section{Differentiation zone/stage}

There was no clear distinction between this and the proliferation zone, and they tended to overlap each other to some extent. During this stage, the inner enamel epithelial cells were differentiating into ameloblasts effectively, but the enamel matrix was not yet formed at all. The stellate reticulum began to decrease suddenly in quantity to as few as only one or two layers.

The inner enamel epithelial cells (Fig. 2-II), facing a thin layer of predentin, became higher $(25 \sim 40 \mu \mathrm{m})$ with ovoid nuclei basally, and the intercellular spaces became narrower. The intercellular junction consisted of basal and distal terminal bars with a few desmosomes, and terminal webs were visible on both sides (Figs. 7,8 ). The basal web was more distinct than the distal one (Fig. 7). Numerous basal cytoplasmic processes projected into the stratum intermedium, and the cytoplasm of this side also did so in a cone-like fashion (Fig. 8). The distal cytoplasmic processes were pushed out into the predentin and eventually became so numerous that the basement membrane was no longer visible, and the microfilaments during the proliferation stage started to become related to the collagenous fibers of the predentin (Fig. 9). The following changes were observed in the cytoplasm as the process of differentiation progressed steadily. The mitochondria developing abundantly shifted from the distal end to the cone-like projection mentioned above (Figs. 2-II, 8). The r-ER, showing a tubular profile,

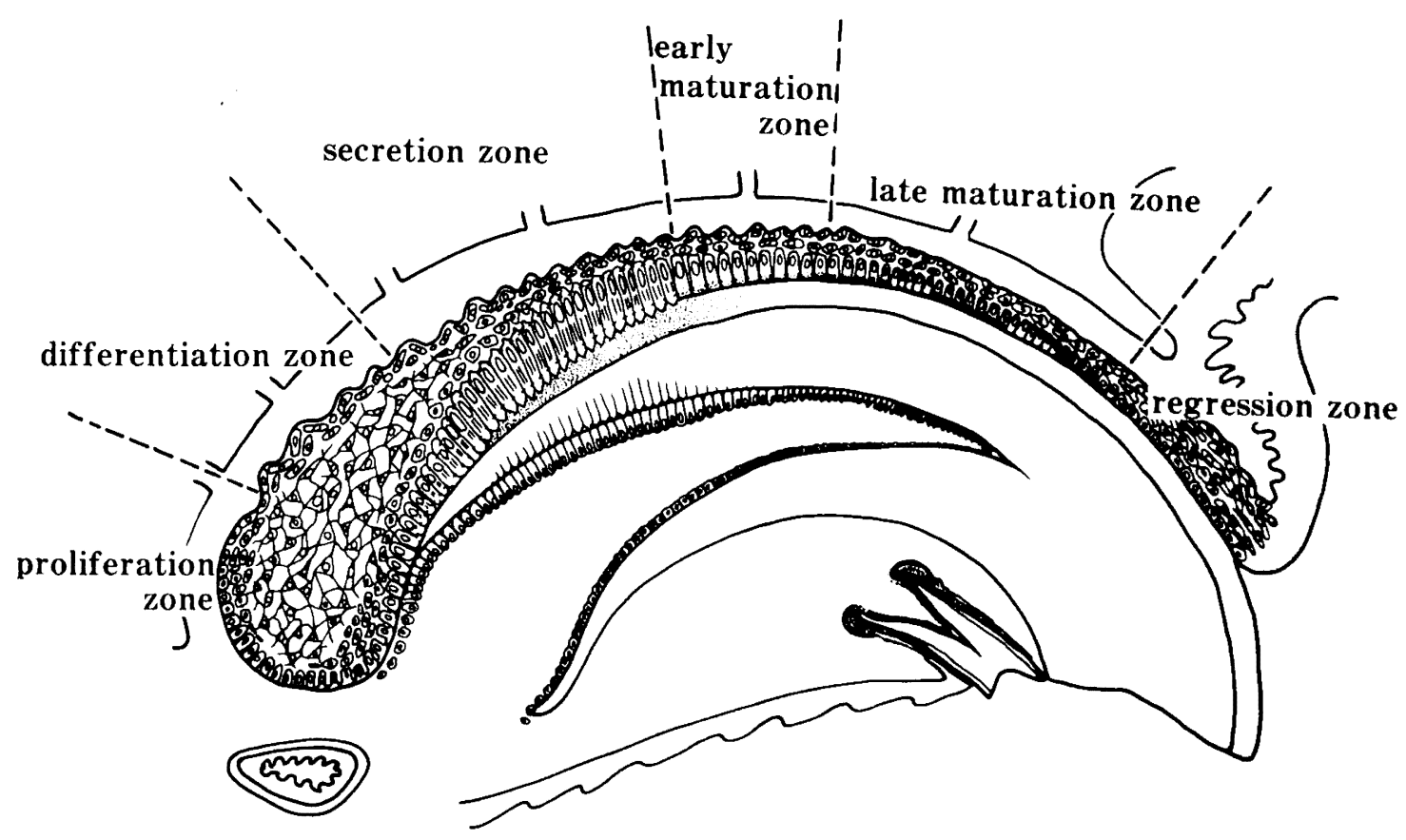

Fig. 1. Schematic, six zones/stages of the ultrastructural changes of all cells composing the enamel organ of the rabbit major incisor. 

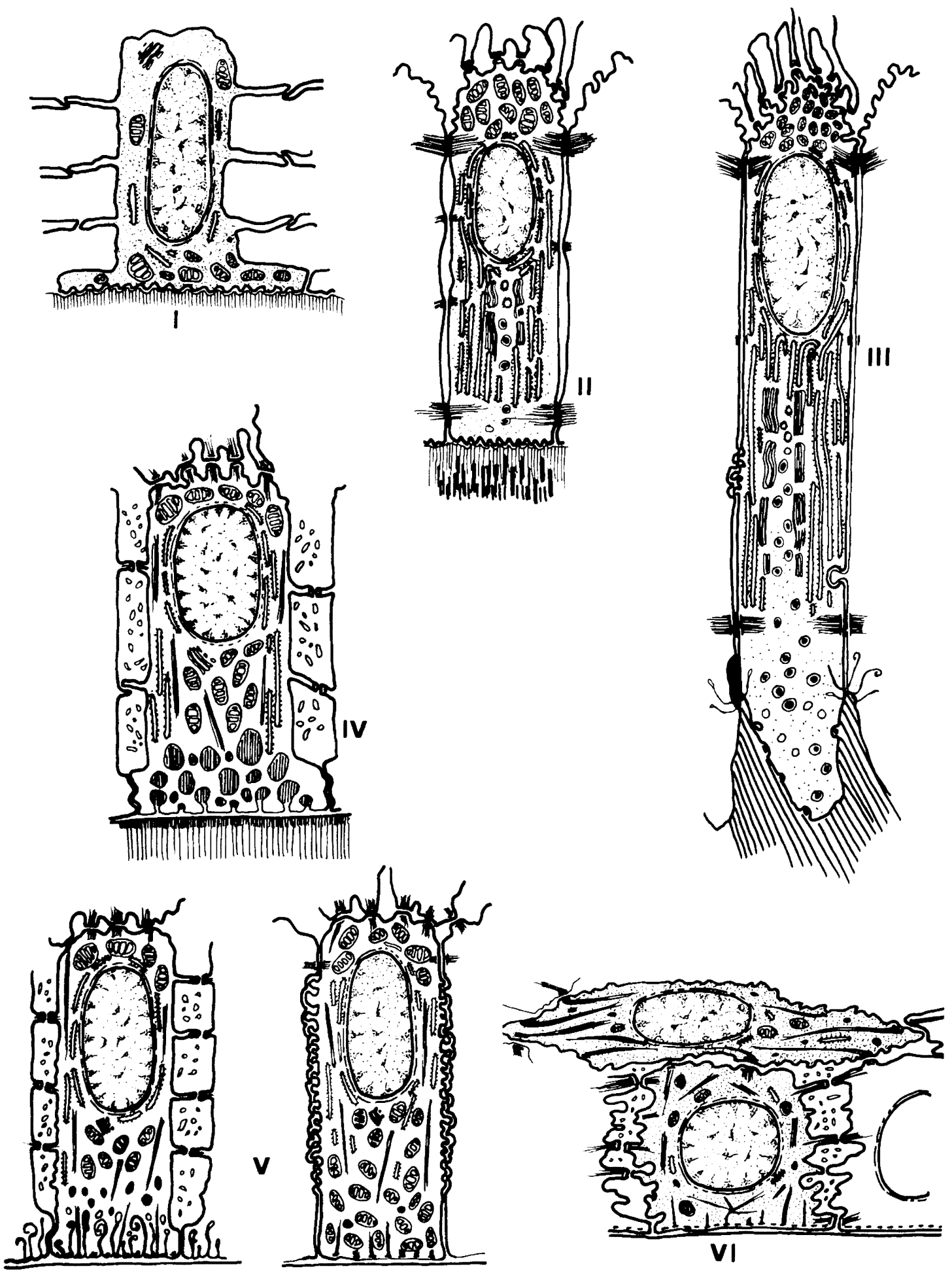

Fig. 2. Ultrastructural changes of the inner enamel epithelial cell (ameloblast) through six zones/ stages. The basal side of the ameloblast is above, and the distal side below in all figures. 
increased in length and amount, and arranged itself around the nucleous towards the distal terminal web along the cell axis (Figs. 2-II, 7). The Golgi apparatus elaborated on the distal side. A long Golgi area (about $3 \mu \mathrm{m}$ in length) was formed in the center of the r-ER, in which presecretory and secretory granules were observed. The cytoplasm distal to the distal terminal web looked clear due to the absence of other major organelles, but with only a few secretory granules and vacuoles (Figs. 7, 9).

The cells of the stratum intermedium, being cuboidal, were arranged in one or two layers. Although similar to that at the previous stage, the cytoplasm was increased in quantity, while the intercellular spaces were reduced with many microvilli. The cellular processes connecting with the inner enamel epithelium by the desmosomes were rather longer (Fig. 8). The Golgi apparatus was situated near the nucleus, and many mitochondria, free ribosomes, a few vacuoles, the dense body and the r-ER were scattered throughout the cytoplasm. The mitochondria, however, began to move towards the inner enamel epithelium, approaching closer to the secretion zone.

The cytoplasmic organelles of the stellate reticulum were similar to those in the previous stage, but the mitochondria and free ribosomes were found in the long cellular processes.

The outer enamel epithelial cells appeared flattened as in the previous stage (Fig. 10). Between the intercellular processes, numerous desmosomes were present and microvilli were located in the intercellular spaces (Fig. 10). This epithelium built up irregular undulations, between the ridges of which blood capillaries were found (Fig. 10). These vessels appeared to be fenestrated in the late part of this stage. The dense bodies, vacuoles and free ribosomes scattered in the cytoplasm increased in quantity, and tonofibrils ran along the cell axis. Many mitochondria were located on the capillary side, also a little r-ER and Golgi apparatus were present around or near the nucleus.

\section{Secretion zone/stage}

The ameloblasts became extremely increased in their height $(60 \sim 70 \mu \mathrm{m})$. The enamel matrix began to deposit up to the final thickness (about $72 \mu \mathrm{m}$ ). The stellate reticulum was still decreased in quantity so that the stratum intermedium was in direct contact with the outer enamel epithelium, of which the undulations became prominent. The capillaries in it became closer to the ameloblasts.

The nucleus of the ameloblasts was also elongated like the cell body, being located basally (Figs. 2-III, 11). The intercellular spaces became narrower except in the area with the microvilli, and the intercellular junction contained the desmosomes, the bulb-type junction and the terminal bar of both sides. Both basal and distal terminal webs were still present (Figs. 11, 12). In cross sections of these webs, the basal web consisted of numerous tonofilaments form. ing a shelf-like structure in order to maintain a large, free space in the center of the ameloblast (Fig. 18). Also, the distal web revealed a similar structure to the above, but the bundles of the tonofilaments (tonofibril) made a network at right angles to the cell axis in its center (Fig. 17).

As the enamel matrix was secreting, the Tomes' process was elongated in a coneshaped fashion to $7 \mu \mathrm{m}$ at longest ( $3 \mu \mathrm{m}$ in the proximal part and $4 \mu \mathrm{m}$ in the distal part) (Figs. 12, 13). The basal cone-like projection and many basal cytoplasmic processes were more developed than in the previous stage (Fig. 11). The spherical mitochondria at this stage were concentrated basally, but did not exist at all distal to the 
necleus. On the other hand, the well-developed Golgi apparatus was located in a distal juxtanuclear position (Fig. 14), and the Golgi lamellae were arranged longitudinally in four or five layers (Fig. 15). In cross sections of the Golgi apparatus, the trans side of the semicircular lamella faced the same side of the opposite lamella (Fig. 16). Within the Golgi area, presecretory and secretory granules were found richly towards the distal side (Figs. 14, 15). Furthermore, abundant tubular r-ER wrapped the nucleus, being arranged longitudinally towards the distal terminal web (Figs. 12, 14). Since no organelles apart from secretory granules and vacuoles were located distal to the distal web, the Tomes' process appeared rather clear (Figs. 12, 13). Morphological differences between the proximal and distal parts of the process were not observed (Fig. 12). In the distal part, however, a membrane fusion system was identified between the coated membrane of the secretory granule and the cytoplasmic membrane.

Just in the late part of this stage, stippled materials were found to some extent between the Tomes' processes. There were a few granular ameloblasts (Kallenbach 1972), in which the Golgi apparatus had disappeared and a little r-ER occured in the periphery of the cell.

The cells of the stratum intermedium extended their cellular processes, being particularly longer towards the ameloblasts, with many desmosomes between adjacent cells (Fig. 11). In the section between this stratum and the ameloblasts, the longer processes surrounded the basal cone-like projections of the ameloblasts and formed a sort of network connecting with the adjacent processes setting the projection in its mesh (Fig. 18). However, the longer processes never advanced distally beyond the basal terminal web. The Golgi apparatus was located juxtanuclearly, and mitochon- dria occurred richly on the ameloblast side (Fig. 11).

In the cells of the stellate reticulum, the mitochondria were extremely increased, differing from those of the previous stage, but the cells were similar in other organelles.

The outer enamel epithelial cells appeared to form complicated ridges, between which fenestrated capillaries were set (Fig. 19). The cellular processes, in particular, proceeded towards the basement membrane surrounding the enamel organ and expanded in a table-shaped fashion to produce uncontinuously diaphragmatic structures (Fig. 19). Eventually, owing to such structures, only the membrane in places was visible between the intercellular spaces of the outer enamel epithelium and the surrounding connective tissue (Fig. 19). Numbers of the mitochondria were concentrated on the capillary side, and many tonofibrils were arranged along the cell axis and continued to the desmosomes. The other organelles were generally similar to those in the previous stage.

IV. Early maturation zone/stage

The enamel calcification proceeded throughout this zone. The ameloblasts suddenly decreased in height, the stellate reticulum became invisible from the middle of the previous stage, and the stratum intermedium did not differ from the outer enamel epithelium, being designated as papillary cells.

The nucleus of the ameloblasts was located basally (Fig. 2-IV). However, the features of the ameloblasts became extremely altered from the late part of the previous stage, reflecting the functional change to maturation, as follows. The basal cone-like projection was reduced in size, but the basal cytoplasmic process was still present (Fig. 20). The basal terminal bar and web gradually disappeared. The distal bar still existed, not in the form of the 
terminal web (Fig. 21). The intercellular spaces become wider, the microvilli extended into the spaces and the desmosomes were formed between the adjacent cells (Fig. 21). The Tomes' process disappeared, and a liner was formed on the distal surface by condensation of the matrix (Fig. 21). Matrix absorption occurred as follows. The liner accompanied by the cytoplasmic membrane was taken into the cell site like a spike up to the mitochondria located distal to the nucleus (Figs. 21, 22). The spike-like invasions became prominent and grew into so-called infoldings. The Golgi apparatus and r-ER were suddenly reduced, becoming scarce in the perinuclear and pericellular portions. The mitochondria located basal to the nucleus in the previous stage were observed not only basally but also distally (Figs. 19, 21). The former were smaller, and the latter larger but never seen within the infoldings. From the desmosome of the basal site, fine tonofibrils extended through the nuclear sides, concentrating into rather thicker ones, and ran up to the distal site, where they again spread into finer tonofibrils (Fig. 22). At the beginning of this stage, they were connected with the distal terminal bar, and afterwards with the half desmosome at the top of the infolding in the subsequent late maturation zone.

Fenestrated capillaries invaded between the papillary cells which were arranged two or three cell thick at the base of the ameloblasts (Fig. 20). Many cellular processes and microvilli extended between the intercellular spaces, and desmosomes were formed between the adjacent cells or ameloblasts (Fig. 20). Especially long processes directed towards the ameloblasts did not exist, while the uncontinuously diaphragmatic structures formed by the cellular processes directed towards the surrounding tissue, still remained (Fig. 20). Abundant microvilli were present between the papillary cells and the basement membrane appearing as a wavy course (Fig. 20). In the papillary cells, numbers of spherical mitochondria were concentrated both in the ameloblasts and the capillary side. The Golgi apparatus was located juxtanuclearly, and a little r-ER, dense bodies and free ribosomes were scattered in the cytoplasm. The tonofibrils were arranged along the cell axis to connect with the desmosomes.

\section{Late maturation zone/stage}

No distinct boundary area was observed between this and the previous zone, although this zone occupied the widest territory. The height of the ameloblasts (20 30 $\mu \mathrm{m})$ became much shorter, presenting a short-cylindrical shape, and the papillary cells as well became flattened, decreasing their layers into only a flattened one or two. Invasion of capillaries as seen in the previous zone was no longer observed, but they were situated very close to the flattened layer.

Two types of ameloblast were now found. One was the so-called ruffle-ended ameloblast (RA), equipped with a wide intercellular space and an infolding in the distal end (Figs. 2-V, 23). The other was the so-called smooth-ended ameloblast (SA) without any such equipment (Figs. 2-V, 24). In this zone, RA bands and SA bands alternated three or four times, and between both bands two types of transitional cells, termed R-SA and S-RA, were present. The overall arrangement was: $\mathrm{RA} \rightarrow \mathrm{R}-\mathrm{SA} \rightarrow \mathrm{SA} \rightarrow$ $S-R A \rightarrow R A$, although the R-SA band was long, and the S-RA band short.

The RA nucleus, being ovoid, was located basally (Fig. 23). Desmosomes existed between adjacent cells and the terminal bar was seen only in the distal site (Fig. 23). The infolding was not large but more complicated (Figs. 23, 25). Accordingly, many vacuole-like structures, which were considered to be cross-sectioned infoldings, and dense granules of various sizes with a coated membrane, were observed around the infold. 
ings (Fig. 25). A dense layer just like a basement membrane, and half desmosomes were present in the cytoplasmic membrane touching the enamel (Figs. 23, 25). The basal site was flattened due to disappearance of the cytoplasmic process. The mitochondria were scattered both basal and distal to the nucleus, but not in the infoldings (Fig. 25). The Golgi apparatus had begun to degenerate, and short tubular r-ER existed along the cell axis and around the nucleus. The tonofibrils left the basal desmosomes to join the distal ones and the half desmosomes of the infoldings.

The location of the SA nucleus was similar to that of the RA (Figs. 2-V, 24). The numbers of desmosomes of the SA were less than those of the RA, and the terminal bar did not exist. The mitochondria of the SA were concentrated up to the distal end at least, but few vacuoles and dense granules were seen (Figs. 24, 26). The r-ER was slightly increased, but the other organelles and basement membrane were similar to those of the RA. The tonofibrils in the SA, however, joined the desmosomes of the basal site from the half desmosomes of the distal site, but not the cell wall (Fig. 2-V). Between the RA and SA bands, basal desmosomes and the distal terminal bar were present, but the former were short in number and the latter was indistinct in form. Such transitional types of ameloblasts were worthy of the name, revealing various kinds of intermediate features between the RA and $\mathrm{SA}$.

The papillary cells were flattened, with narrower intercellular spaces, in that many cellular processes and microvilli existed, and the desmosomes were widened and increased (Fig. 23). The table-like diaphragmatic structures observed until the previous stage had now disappeared, so that the space between the papillary cells and the basement membrane did not exist (Fig. 23). Undulations were seen in the basement membrane, and half desmosomes were found on the tops of them. The r-ER and free ribosomes were scattered in the cytoplasm. The Golgi apparatus was located juxtanuclearly, and thick tonofibrils numbering more than those in the previous stage, were arranged along the cell axis and joined the desmosomes.

\section{Regression zone/stage}

Both the ameloblasts and the papillary cells were not cuboid, and more flattened near the inner marginal epithelium, without any distinct border between them. In particular, the latter were much flattened and elongated tooth-axially (Figs. 27, 28).

The cytoplasm of the ameloblasts was in general dense with a flattened nucleus (Figs. 2-VI, 27, 28). The cellular processes and microvilli in the narrow intercellular space were connected with adjacent ones by several desmosomes (Figs. 27, 28). However, wide desmosomes were observed between the ameloblasts and the papillary cells (Figs. 27, 28). Neither the terminal bar nor infolding was observed in the distal sites, so that the distal end touching the enamel was flattened, where half desmosomes and continuous basement membrane distal to it were observed (Figs. 27, 28). The increased tonofibrils in this stage were attached to the basal desmosomes to form irregular networks while the distal ones joined those on the lateral side and the distal half desmosomes (Figs. 27, 28). Many dense bodies, a few mitochondria and vacuoles were present, but $r$-ER and Golgi apparatus were not found in the transitional cell band.

The papillary cells were elongated and flattened in the tooth-axis. The cellular processes were reduced on connected with adjacent cells by desmosomes in a wide area (Figs. 27, 28). The intercellular space became narrower and the microvilli were decreased. Those cells which faced the surrounding connective tissue and the 
basement membrane and changed to the inner marginal epithelium, revealed slightundulations, on the tops of which were prominent half desmosomes (Figs. 27, 28). A few dense bodies, mitochondria and r-ER were seen, but no Golgi apparatus (Figs. 27, 28). Leucocytes were sometimes present between the papillary cells. There were strange cells, considered to be degenerated, with an ovoid nucleus, cytoplasm of higher density but of small amount, no tonofilbrils, no desmosomes between adjacent cells, and no cytoplasmic organelles.

\section{Discussion}

Differences in cellular changes through three stages of the enamel organ of the upper incisor of the rabbit have been described at the light microscopic level by Suga (1959). Such morphological changes have also been investigated in the rat, mouse and hamster under both the light and electron microscope by Pindborg et al. (1959) and others. Strangely, no ultrastructural descriptions have yet been made for the rabbit incisor.

The developmental process of the enamel organ of the rabbit incisor was classified into six zones/stages in the present paper. Each stage was compared with that of Kallenbach et al. (1965) in the rat incisor as follows: the early stage of the postsecretion zone of the rat may correspond to the early maturation zone of the rabbit, an the late postsecretion zone and following pigmentation zone of the rat to the late maturation zone of the rabbit, respectively. The late maturation of the rabbit appeared as an alternation of the ruffle-ended ameloblasts (RA) and smooth-ended ameloblasts (SA), without the presence of the iron-pigmentation as seen in the rat. Referring to such features, Josephsen et al. (1977) attached greater importance to the two types of ameloblasts than the appearance of iron-pigment, and indicated the region of ameloblast modulation. The present author agrees with his analysis.

\section{Proliferation zone}

The inner enamel epithelial cell is considered to be on the way to the following stage based on the morphology of the nucleus and intracellular organelles, as described for the rat by Reith (1967), Ichijyo (1969), Kallenbach (1971) and Katchburian et al. (1972). The distal location of the mitochondria may indicate that the metabolic pathway exists in the site of the dental papillary cell, as stated by Frank et al. (1967) and the above investigators. It was distinguishable in the rabbit since the intercellular spaces were widened in this epithelium, where no attachment was observed between the cellular processes. Although such findings differ from those of other species as reported in other papers, they did represent a favorable feature for the supply route from the site of the dental papillary cells.

Microfilaments running from the basement membrane towards the dental papilla at right angles have been observed in the cat and man (Frank et al. 1967) and in the rat (Kallenbach 1971, Reith 1967 and Takuma 1967). Frank et al. and Kallenbach stated that the microfilaments determined the course and arrangement of the collagen fibers in the predentin. These would contribute to the formation of the predentin, and are retained by the half desmosomelike structures at the distal end.

\section{Differentiation zone}

The inner enamel epithelial cell differentiates into the ameloblast within this zone, as indicated in the rat by Reith (1967), Ichijyo (1969) and Moe (1971). Furthermore, this histodifferentiation in the rat was subdivided into six stages by Kallenbach (1971). Although differentiated cells do not present a regular and similar arrange- 
ment and form, they finally grow into ameloblasts of the same form as indicated by him. Differentiation of these cells in the rabbit can be summarized as follows. The basal cytoplasm of the inner enamel epithelial cell develops into the basal cytoplasmic process, and then projects in conelike fashion with the mitochondria. The stellate reticulum decreases in quantity. The cellular processes of the stratum intermedium markedly elongate towards the inner enamel epithelium. The outer enamel epithelium appears as irregular undulations, between the ridges of which fenestrated capillaries intervene. According to such changes, the latter epithelium and capillaries become close to the stratum intermedium. The mitochondria and cytoplasm suddenly increase in both epithelial cells. Based on these findings, the metabolic pathway may switch from the site of the dental papillary cell during the previous stage to the site of the opposite capillaries within the connective tissue surrounding the enamel organ.

In the inner enamel epithelial cell of the rabbit, the area distal to the terminal bar is found as a clear zone because of the few vacuoles and secretory granules. This corresponds to the clear distal area of the preodontoblast and young odontoblast in the rat proposed by Takuma et al. (1971). This area may be in preparation for the Tomes' process. However, Warshawsky (1966) concluded that the secretory granule was not formed at this stage because the enamel matrix protein was synthesized in the r-ER to be reserved in the form of the intracisternal granule.

The differences between the microfilaments of the inner enamel epithelial cells and the collagenous fibers of the predentin cease to remain obvious. Such features may contribute to the dentinoenamel junction.

In the outer enamel epithelial cell, the tonofibrils appear as the cytoskeleton, which may act as a kind of trabeculae to the set-in of the capillaries. A sudden increase in the microvilli, the pinocytotic vesicle and mitochondria may suggest the introduction of metabolic materials into the cell as shown by Ichijyo (1969) in the rat.

\section{Secretion zone}

Many investigations have been made of the inner enamel epithelium at this stage, used as the ameloblast in certain mammals under the light and electron microscope.

Since Watson (1960) suggested "the extracellular nature" whereby secretory granules were extruded for the enamel matrix formation in the rat, many workers have agreed with his suggestion in other species, including also the rabbit as described in this paper. The intracellular synthesis of the secretory granules may be summarized as follows. The mitochondria, being the energy-supplying source, are concentrated on the basal side of the nucleus, and the $r-E R$ is arranged around the nucleus and along the cell axis up to the distal terminal web for the purpose of protein-synthesis. The Golgi apparatus is located in the center of the $r-E R$, where the presecretory granules are condensed and packed against the secretory granules, which finally move towards the Tomes' process in the distal site. These structures have been described by Warshawsky et al. (1966), Frank (1970), Weinstock et al. $(1970,1971)$ and Kallenbach (1972). Elwood et al. (1968) were skeptical about the arrangement of the organelles in the rat ameloblast in the enamel matrix formation as follows. The product of the protein-secreting glandular cell was formed in the endoplasmic reticulum basally, "packaged" in single-membrane vesicles at the level of the Golgi apparatus, and extruded from the apical end of the cell. The r-ER of the ameloblast, on the other hand, rather than the Golgi region, lay closest to the extrusive end of the cell, so that this did not exhibit an accumulative secretory phase. If several cells in a glandular 
acinus could be compared to one ameloblast, it would be possible to envisage a pattern in which the Golgi apparatus is located in the center and the r-ER in a circle. Such a situation may be reasonable for abundant formation of secretory granules in a short period.

The distal terminal web was found as a shelf-like structure pericellularly in previous studies: Kallenbach et al. (1965), Reith (1960, 1967) and Frank (1970) in the rat, Grant et al. (1968) in the mouse, and Rönnholm (1962) in man. In the distal terminal web of the rabbit, a network of tonofibrils may prevent movement of the organelles into the Tomes' process and may permit only secretory granules which would be extruded rapidly to pass. Accordingly, the Tomes' processes appear to be clear. Furthermore, Kallenbach (1972) stated that the granular ameloblast may be caused by a block in the transference from the intracisternal granule of the r-ER to the Golgi apparatus. The present author, however, is of opinion that the granular ameloblasts may be formed by the accumulation of secretory granules, which are too large to pass through the network. Accordingly, the granular ameloblasts in nature are not visible in the subsequent stage because of the disappearance of the network. The basal terminal web forms the shelflike structure without the network in order to maintain freely active function of the mitochondria. Such features have been reported in man by Rönnholm (1962) and in the rat by Grant et al. (1968) and Ichijyo (1969).

Stippled material between the Tomes' processes and between the ameloblasts has been described in the rat incisor and molar by Watson (1960) and Fernhead (1960, 1961), in man by Rönnholm, in cattle by Travis et al. (1964), and in the rat incisor by Ichijyo. In the present study, such stippled material was seldom seen in the upper major incisor of the rabbit, although it was found at the secretory end of the ameloblast at this late stage. Owing to the slow secretion in the early stage, the secretory end of the ameloblast at this late stage. Owing to the slow secretion in the early stage, the secretory product may change immediately to fibrous matrix, but may appear as precursor of the matrix owing to the rapid secretion in this late stage. Accordingly, the stippled material may be invisible in the former period, but visible to some extent in the latter.

As regards the metabolic pathway of the ameloblast, the present author offers the following suggestion. The cone-like projection of the ameloblast and the basal cytoplasmic process develop to the utmost extent and are surrounded by long cellular processes of the stratum intermedium. Such wide contacts between them should render the pathway larger and more reasonable, while the deep invasion of the fenestrated capillaries becomes closer to the stratum intermedium, with extension of the cellular process of the outer enamel epithelium. Finally, the basement membrane comes in direct contact with the intercellular spaces of the outer enamel epithelium in many places, not being interposed by any other structures. These features are more favorable for the metabolic pathway of the ameloblast than those at the previous stage.

\section{Early maturation zone}

The ameloblasts now become reduced in height with the Tomes' process not visible, representing the so-called short ameloblast of the rat named by Wassermann (1944). No report, however, has yet been made for the rabbit incisor, but for the rat incisor by Kallenbach (1968, 1974), Reith (1961, 1963), Elwood et al. (1968), Kurahashi et al. (1969), Moe (1971) and Smith (1979) and molar by Reith (1970). This stage in the rabbit was found to be rather 
shorter, about two-thirds of that in the rat.

Morphological changes to the ameloblast have begun in the late part of the previous stage. The mitochondria are concentrated distally and basally. The distal mitochondria may be related to the calcification as pointed out by Iwakura (1973), and matrix absorption as pointed out by Wassermann (1944) in this stage. The basal mitochondria carrying over from the previous stage may be related to the pathways of calcium and energy.

The present author makes the following analysis concerning the matrix absorption. A liner which represents condensation of the matrix, gradually develops into the infoldings, and is found to be irregular and dense near the absorption site, but finally disappears at the distal mitochondrial level. The infoldings with increased surface may be convenient for a major amount of calcium secretion in a short period.

Several tonofibrils are found in the center of the ameloblast, although Kallenbach et al. $(1965,1968)$ enumerated one to be a cytoskeleton. The appearance of the tonofibrils may be related to the junctional status between the ameloblast and the matrix being calcified rapidly. The increase of the tonofibrils in the papillary cells may occur as a result of a resistance against mastication.

In this paper, both the cells of the stratum intermedium and the outer enamel epithelium are taken as papillary cells, as did Williams (1896) in the rat. However, they are not seen as obvious papillae in the rabbit.

Part of the capillaries contacting the papillary cells may have a passage to their intercellular spaces, only interposing the basement membrane. This suggests that material transport may be carried out extracellularly. Also, a feature such as the so-called transport epithelium is under- standable from the concentration of mitochondria in both the ameloblast and capillary sides.

\section{Late maturation zone}

The ameloblast at this stage has been variously designated as follows: a "short" (Wassermann 1944), "transporting" (Reith 1967), "postsecretory" (Grant et al. 1968), "maturation" (Reith 1970), and "adsorptive" (Warshawsky 1971, 1974) ameloblast, and absorptive amelocyte (Moe 1971). In the rat and hamster among the Rodentia, two types of ameloblasts have been reported as the RA and SA by Josephsen et al. (1977). These two types were described in the rabbit incisor based on light microscopic and histochemical studies by Suga (1959, 1964). The cells considered in this paper are cylindrical, shorter than those of the rat, but do not contain iron-pigment granules. Since Josephsen et al. clarified the differences between the two types under the electron microscope, the present author undertook detailed observations of the rabbit major incisor at the same order of magnification. The mitochondria of the RA exceed those of the SA in the rat incisor, but in the rabbit incisor no differences are observed between the cells. The Golgi apparatus is more distinct and well-developed in the SA. Takano et al. $(1980,1982)$ attempted to discuss the functional differences based on histochemical procedures. Even so, morphological differences between the two types of ameloblast are not distinct in the rabbit. This may indicate a rather short expiration of the cell activity. Josephsen et al. suggested that the intercellular junction was found to be the terminal bar distally in the RA, but basally in the SA, and changes in these attachments might contribute to the material transport. However, the SA of the rabbit had basal desmosomes with narrow intercellular spaces where microvilli were concentrated. This indicates that the material transport may not 
be active.

\section{Regression zone}

Since the ameloblasts become transitional to the junctional epithelium, they have been termed reduced ameloblasts. Such features have been reported in the rat incisor (Reith 1961, Kallenbach 1970 and Yamada et al. 1982), in man (Listgarten 1966 and Shimono et al. 1981) and in the kitten (Sasaki et al. 1981). The basement membrane on the distal end of the ameloblast from the early maturation zone, still has many half desmosomes. They were not similar to those described by Kallenbach, but more indistinct and smaller in extent than those of the basal side of the enamel organ.

From the well-developed intercellular junctions and cytoskeleton, the reduced ameloblasts and papillary cells gradually but steadily approach junctional epithelial cells. Although Kallenbach (1970) reported "light cells" between the papillary cells, dark and dense cells which appeared to be degenerated, were noted in the present study.

\section{Acknowledgements}

The author wishes to express his sincere thanks to Professor Y. Ohta for critically reading the manuscript, and also to Assistant Professor T. Tokioka and the staff members of the Department of Anatomy for their invaluable assistance.

\section{Literature Cited}

1) Elwood, W. K. and M. H. Bernstein: The ultrastructure of the enamel organ related to enamel formation. Am. J. Anat., 122: 73-94, 1968.

2) Fearnhead, R. W.: Mineralization of rat enamel. Nature, Lond., 188: 509-510, 1960.

3) Idem: Secretory products of ameloblasts. In Electron Microscopy in Anatomy, 241260. Edwasrd Arnold, London, 1961.

4) Frank, R. M. and J. Nalbandian: Ultrastruc- ture of amelogenesis. Structural and chemical organization of teeth, Vol. I, 399-466. Academic Press, New York, 1967.

5) Frank, R. M.: Autoradiographie quantitative de l'amélogenèse en microscopie électronique a l'aide de la proline tritée chez le chat. Archs. oral Biol., 15: 569-581, 1970.

6) Grant, P. R. and J. Nalbandian: Observation on the ultrastructure of ameloblasts with special reference to the Golgi complex and related components. J. Ultrastruct. Res., 23: 427-443, 1968.

7) Ichijyo, T.: Ultrastructural research of the morphological changes in the developing ameloblast and the formation of the enamel matrix. Hard Tissue Research, 286-342. Ishiyaku, Tokyo, 1969 (in Japanese).

8) Iwakura, M.: The mechanism of bone calcification - the calcium transport of the hard tissue forming cells. Protein, Nucleic Acid and Enzyme, 18: 1012-1017, 1973 (in Japanese).

9) Josephsen, K. and A. Fejerskov: Ameloblast modulation in the maturation zone of the rat incisor enamel organ. $\mathbf{A}$ light and electron microscopic study. J. Anat., 124: 45-70, 1977.

10) Kallenbach, E.: Fine structure of rat incisor ameloblasts during enamel maturation. J. Ultrastruct. Res., 22: 90-119, 1968.

11) Idem: Fine structure of rat incisor enamel organ during late pigmentation and regression stages. J. Ultrastruct. Res., 30: 38-63, 1970.

12) Idem: Electron microscopy of the differentiating rat incisor ameloblast. J. Ultrastruct. Res., 35: 508-531, 1971.

13) Idem: Granules in cisternae of the rough endoplasmic reticulum (RER) of preameloblasts and ameloblasts and a possible function of the RER in preameloblasts of rat incisor. J. Ultrastruct. Res., 39: 96-105, 1972.

14) Idem: The fine structure of Tomes' process of rat incisor ameloblasts and its relationship to the elaboration of enamel. Tissue \& Cell, 5: 501-524, 1973.

15) Idem: Fine structure of rat incisor ameloblasts in transition between enamel secretion and maturation stages. Tissue \& Cell, 6: 173 190. 1974.

16) Kallenbach, E., Y. Clermont and C. P. Leblond: The cell web in the ameloblasts of the rat incisor. Anat. Rec., 153: 55-70, 1965.

17) Katchburian, E. and S. J. Holt: Studies on the development of ameloblasts. I. Fine struc- 
ture. J. Cell Sci., 11: 415-447, 1972.

18) Kurahashi, Y. and H. Moe: Electron microscopy of the ameloblast in the maturation stage of the enamel in the rat. Hard Tissue Research, 254-285. Ishiyaku, Tokyo, 1969 (in Japanese).

19) Listgarten, M. A.: Phase-contrast and electron microscopic study of the junction between reduced enamel epithelium and enamel in unerupted human teeth. Archs. oral Biol., 11: 999-1016, 1966.

20) Moe, H.: Morphological changes in the infranuclear portion of the enamel-producing cells during their life cycle. J. Anat., 108: 43-62, 1971.

21) Pindborg, J. J. and J. P. Weinmann: Morphological and functional correlations in the enamel organ of the rat incisor during amelogenesis. Acta anat., 36: 367-381, 1959.

22) Reith, E. J.: The ultrastructure of ameloblasts from the growing end of rat incisors. Archs. oral Biol., 2: 253-262, 1960.

23) Idem: The ultrastructure of ameloblasts during matrix formation and the maturation of enamel. J. Biophys. Biochem. Cytol., 9: 825-839, 1961.

24) Idem: The ultrastructure of ameloblasts during early stages of maturation of enamel. J. Cell Biol., 18: 691-696, 1963.

25) Idem: The early stage of amelogenesis as observed in molar teeth of young rats. J. Ultrastruct. Res., 17: 503-526, 1967.

26) Idem: The stage of amelogenesis as observed in molar teeth of young rats. J. Ultrastruct. Res., 30: 111-151, 1970.

27) Reith, E. J. and V. F. Cotty: The absorptive activity of ameloblasts during maturation of enamel. Anat. Rec., 157: 577-588, 1967.

28) Rönnholm, E.: An electron microscopic study of the amelogenesis in human teeth. I. The fine structure of the ameloblasts. J. Ultrastruct. Res., 6: 229-248, 1962.

29) Sasaki, T., T. Nakagawa, H. Tominaga, T. Kawahara and S. Higashi: Electron microscopy of the junctional epithelium of the kitten gingiva. Bull. Tokyo dent. Coll., 22: 139-149, 1981.

30) Shimono, M., K. Sugiura and T. Yamamura: Intercellular junctions of normal human gingival epithelium. A study using freezefracture. Bull. Tokyo dent. Coll., 22: 223236, 1981.

31) Smith, C. E.: Ameloblast: Secretory and resorptive functions. J. Dent. Res., 58 (Sp. Iss. B): 695-706, 1979.
32) Suga, S.: Amelogenesis. Some histological and histochemical observations. Int. Dent. J., 9: 394-420, 1959.

33) Suga, S.: Mechanism of the enamel formation - maturation. Koku Seibutsu Gaku no Kenkyu, 5: 147-171, 1964 (in Japanese).

34) Takano, Y. and H. Ozawa: Ultrastructural and cytochemical observations on the alternating morphologic changes of the ameloblasts at the stage of enamel maturation. Arch. histol. jap., 43: 385-399, 1980.

35) Takano, Y., M. A. Crenshaw, J. W. Bawden, L. Hammarström and S. Lindskog: The visualization of the patterns of ameloblast modulation by the glyoxal bis (2-hydroxyanil) staining method. J. Dent. Res., 61: (Sp. Iss.): 1580-1586, 1982.

36) Takuma, S.: Ultrastructure of dentinogenesis. Structural and chemical organization of teeth. Vol. I, 325-370. Academic Press, New York, 1967.

37) Takuma, S. and N. Nagai: Ultrastructure of rat odontoblasts in various stages of their development and maturation. Archs. oral Biol., 16: 993-1011, 1971.

38) Travis, D. F. and M. J. Glimcher: The structure and organization of, and the relationship between the organic matrix and the inorganic crystal of embryonic bovine enamel. J. Cell Biol., 23: 447-496, 1964.

39) Warshawsky, H.: Steps in secretion of enamel matrix protein, as shown by electron microscope radioautography of the ameloblasts of rat incisors following tyrosine- $\mathrm{H}^{3}$ injection (abstract). Anat. Rec., 154: 438, 1966.

40) Warshawsky, H. and C. E. Smith: Morphological classification of rat incisor ameloblasts. Anat. Rec., 179: 423-446, 1974.

41) Wassermann, F.: Analysis of the enamel formation in the continuously growing teeth of normal and vitamin $\mathrm{C}$ deficient guinea pig. J. Dent. Res., 23: 463-510, 1944.

42) Watson, M. L.: The extracellular nature of enamel in the rat. J. Biophys. Biochem. Cytol., 7: 489-491, 1960.

43) Weinstock, A.: Cytotoxic effects of puromycin on the Golgi apparatus of pancreatic acinar cells, hepatocytes and ameloblasts. J. Histochem. Cytochem., 18: 875-886, 1970.

44) Weinstock, A. and C. P. Leblond: Elaboration of the matrix glycoprotein of enamel by the secretory ameloblasts of the rat incisors as revealed by radioautography after galactose$\mathrm{H}^{3}$ injection. J. Cell Biol., 51: 26-51, 1971.

45) Williams, J. L.: On the formation and struc- 
ture of dental enamel. Dental Cosmos., 38: 101-127, 1896.

46) Yamada, S., T. Kasai and T. Sato: Electron microscopic study of regeneration of junctional epithelium. Bull. Tokyo dent. Cell., 23: 145-157, 1982.

\section{Abbreviations}

\author{
AB : ameloblast \\ BC : blood capillary \\ BW : basal terminal web \\ D : desmosome \\ DW : distal terminal web \\ E : enamel \\ IE : inner enamel epithelium
}
M : mitochondria
OE : outer enamel epithelium
$P$ : papillary cell
r-ER : rough endoplasmic reticulum
SI : stratum intermedium
SG : secretory granule
TF : tonofibril 


\section{Explanation of Figures}

\section{Plate I}

Fig. 3. The respective cells of the inner enamel epithlium, the statum intermedium, the stellate reticulum (SR) and the dental papilla (DP) in the proliferation zone. The wide intercellular spaces at the distal end are blocked by the cellular processes of the inner enamel epithelial cells. The microfilaments (MF) run from the basement membrane $(\leftrightarrow)$ of the enamel organ towards the dental papilla. The mitochondria of the dental papillary cells are concentrated on the enamel organ side. $\times 5,600$.

Fig. 4. Outer enamel epithelial cells of the proliferation zone. The basement membrane $(\rightarrow)$ is found between this epithelium and the surrounding connective tissue. A few desmosomes are present between the adjacent processes of the epithelial cells, with a few free ribosomes and mitochondria. (FB) fibroblast. $\times 12,000$.

Fig. 5. Basal side of the inner enamel epithelial cell of the proliferation zone. The undeveloped Golgi apparatus (G) is located around the nucleus. $\times 16,000$.

Fig. 6. Distal end of the inner enamel epithelial cell of the proliferation zone. The mitochondria are located distally and the microfilaments (MF) run towards the dental papilla at right angles. ( $\leftarrow)$ basement membrane, (DG) dense granule, (HD) half desmosome. $\times 41,250$. 
Plate I

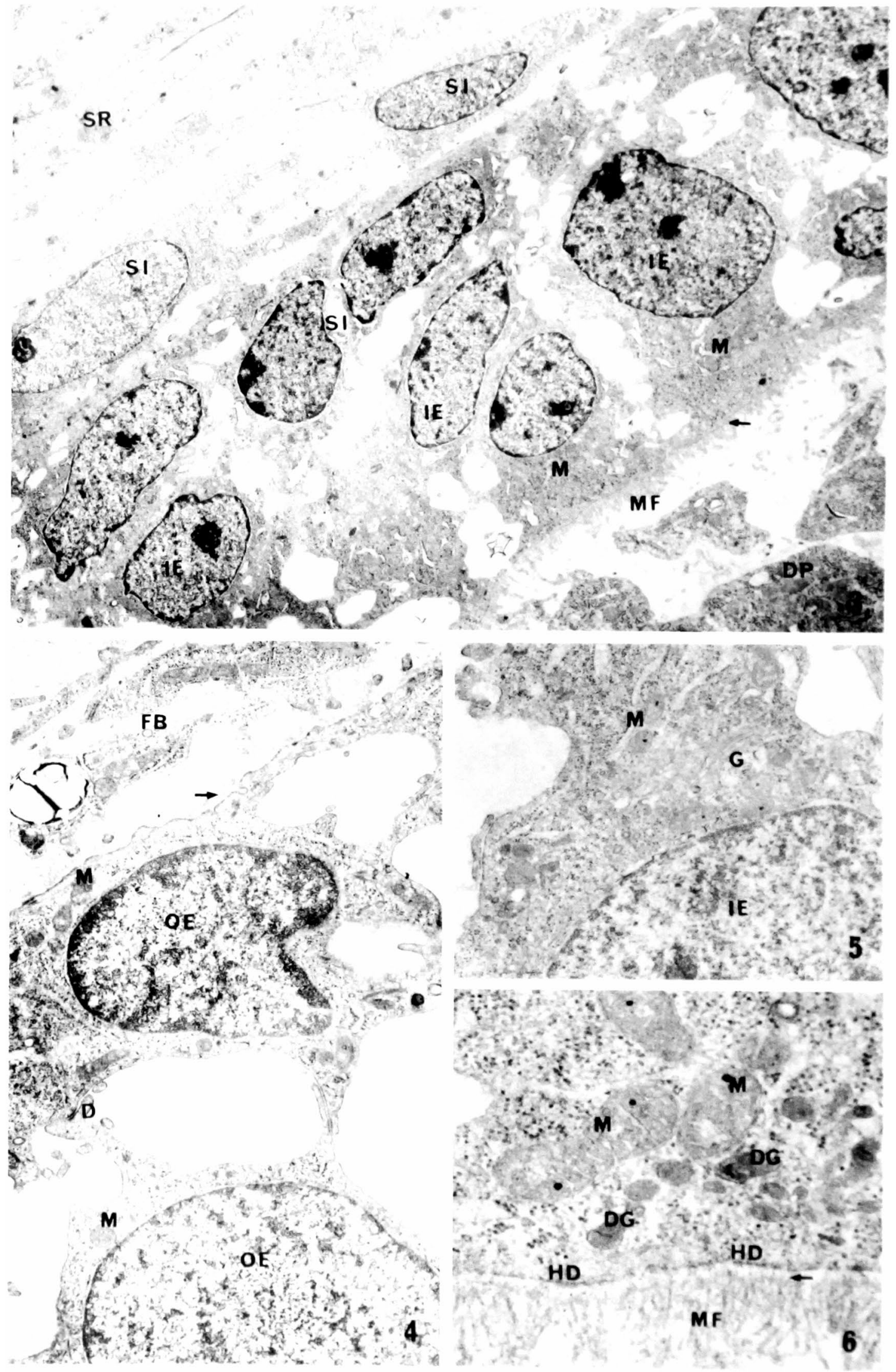




\section{Plate II}

Fig. 7. Distal side of the inner enamel epithelial cells of the differentiation zone. The intercellular spaces are suddenly narrowed. The distal terminal webs are formed, but no major organelles exist beyond the distal ones. (PD) predentin. $\times 3,600$.

Fig. 8. Basal side of the inner enamel epithelial cells of the differentiation zone. The basal terminal webs and many basal cytoplasmic processes (BP) are formed. The processes are connected to the cellular processes of the stratum intermedium cells with desmosomes. The nucleus and mitochondria are located basally. $\times 6,000$.

Fig. 9. Distal end of the inner enamel epithelium of the differentiation zone. As the microfilaments start to become related to the collagenous fibers (CF) of the predentin (PD), the basement membrane is invisible. $\times 20,600$.

Fig. 10. Outer enamel epithelial cells of the differentiation zone. These cells are flattened with irregular undulations, between the ridges of which blood capillaries are found. Many microvilli are seen between the intercellular spaces, and the mitochondria are located on the capillary side. ( $t$ ) basement membrane. $\times 4,120$.

Fig. 11. Basal side of the ameloblasts of the secretion zone. At this site, cone-like projections (CP) are formed with the basal cytoplasmic processes (BP), which are connected to the cellular processes of the stratum intermedium with desmosomes. The mitochondria are present in these projections.

$\times 6,250$.

Fig. 12. Distal side of the ameloblast of the secretion zone. The tubular r-ER which is increased in length and amount is arranged longitudinally, but does not exist distal to the distal terminal webs. (TP) Tomes' process. $\times 8,250$. 


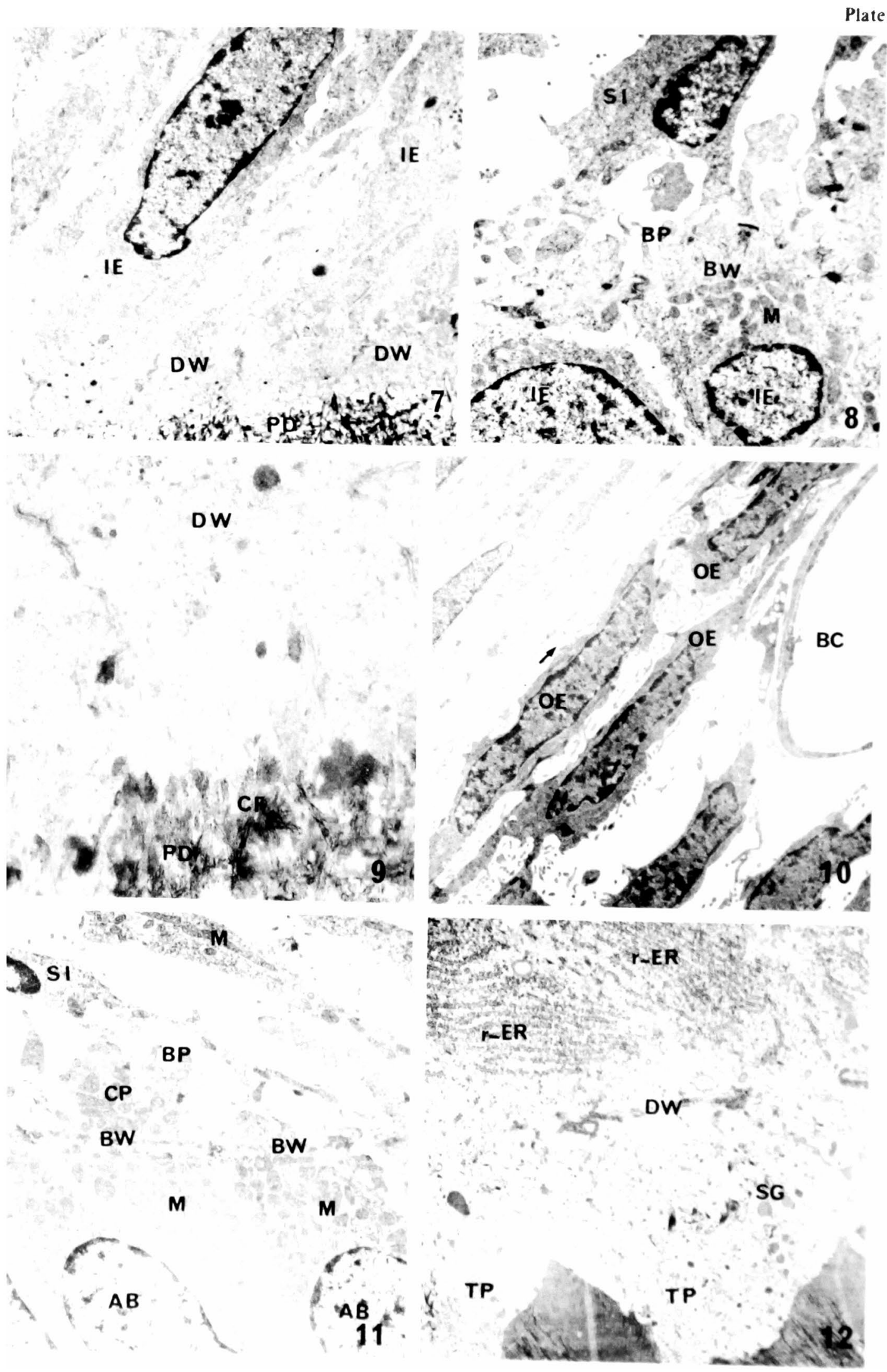




\section{Plate III}

Fig. 13. The Tomes' process of the ameloblast of the secretion zone. Organelles are not present in this process except secretory granules. $(E M)$ enamel matrix. $\times 11,900$.

Fig. 14. Distal area of the nucleus of the ameloblast of the secretion zone. The Golgi apparatus (G) is located cell-axially, as is also the r-ER. $\times 8,630$.

Fig. 15. Longitudinally sectioned Golgi apparatus. $\times 20,000$.

Fig. 16. Cross-sectioned Golgi apparatus. $X 14,000$.

In Fig. 15, the Golgi lamellae (GL) are arranged longitudinally in four or five layers, between which many secretory granules are formed. In Fig. 16, the semicircular lamellae face the same side of the opposite lamellae.

Fig. 17. Cross-sectioned, distal terminal web of the ameloblast of the secretion zone. The shelf-like structure (SL) is formed around the cell wall and the tonofibril network in the cell center. $\times 20,600$. 


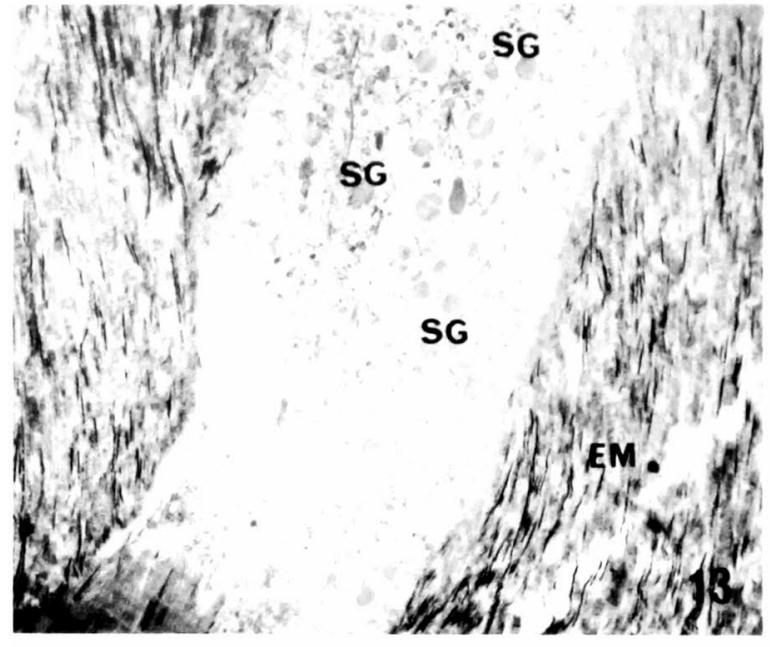

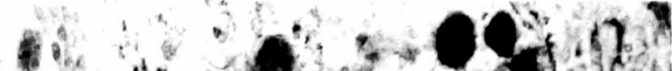

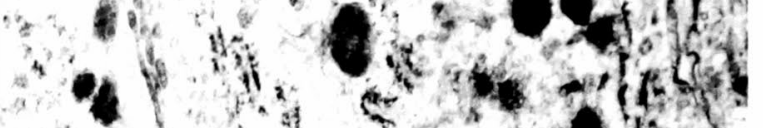

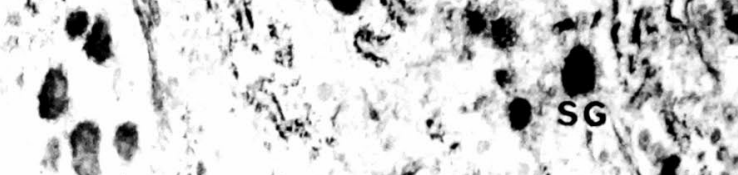
3) SG $1, y$, GL $\quad$ (2)

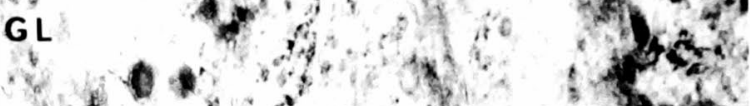
i. $10.0 \mathrm{GL}$ (1) SG 40 का

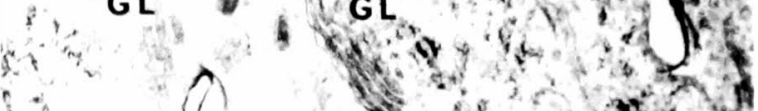

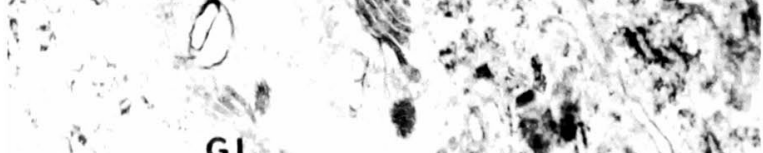

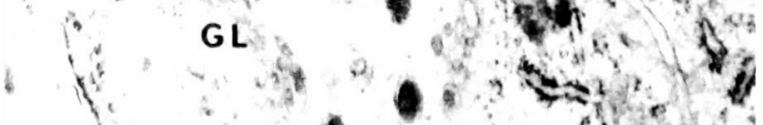
a

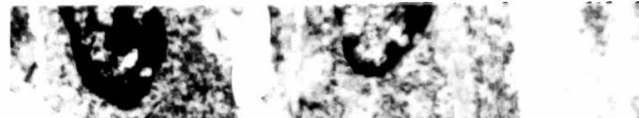
tration

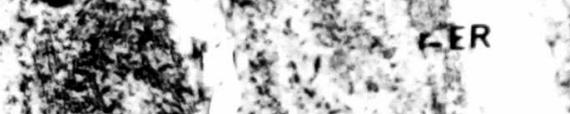

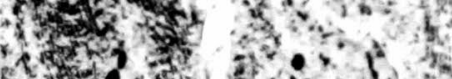

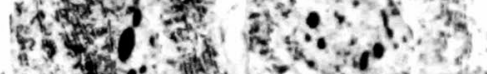

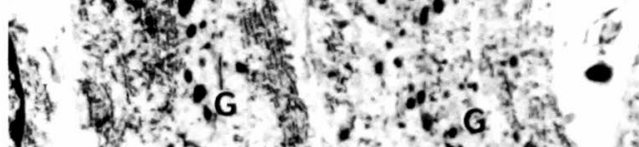

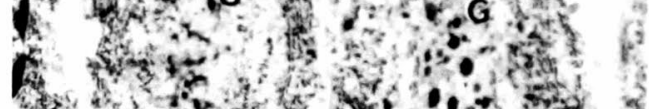

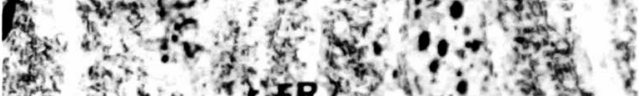
3.

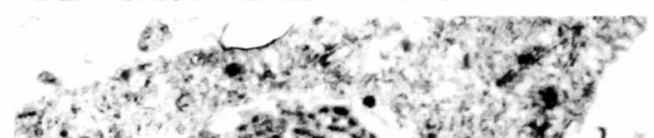

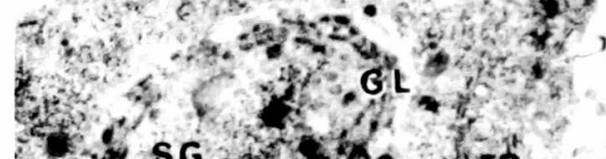

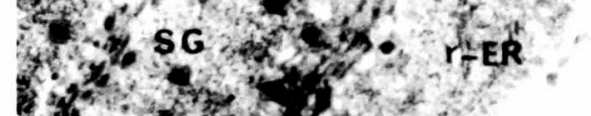

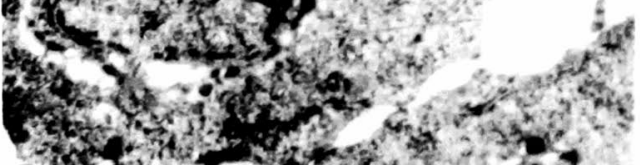
4. is

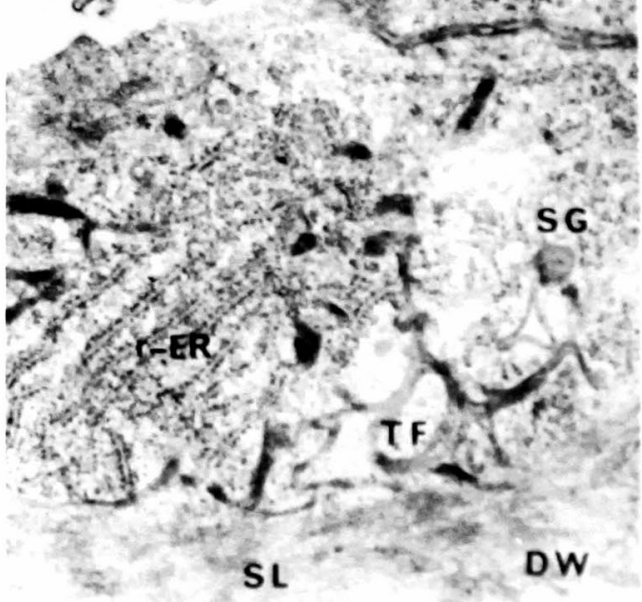




\section{Plate IV}

Fig. 18. Oblique section of the stratum intermedium through the basal side of the ameloblast of the secretion zone. The cone-like projections (CP) of the ameloblast are surrounded by the long processes of the stratum intermedium cells. The tonofibril network as seen in the distal web is not found in the sectioned area of the basal terminal webs. $\times 2,750$.

Fig. 19. Outer enamel epithelial cells of the secretion zone. Uncontinuously diaphragmatic structures of the cellular processes are seen along the basement membrane $(\leftarrow)$ with many microvilli in the intercellular spaces. Blood capillaries exist between the ridges of the undulations of the outer enamel epithelium. $\times 2,750$.

Fig. 20. Papillary cells and the basal side of the ameloblasts of the early maturation zone. The cone-like projections disappear and the intercellular spaces are widened. Blood capillaries invade between the papillae, with many microvilli in the intercellular spaces. $(\leftarrow)$ basement membrane. $\times 3,000$.

Fig. 21. Distal side of the ameloblasts of the early maturation zone. The distal terminal web disappears but the terminal bar (TB) is persistent. The Tomes' process disappears and a liner $(t)$ is formed on the distal surface by condensation of the matrix. Absorption (A) of the matrix occurs. The mitochondria are found both on the basal and distal sides. $\times 9,250$.

Fig. 22. Distal end of the ameloblast of the early maturation zone. The liner ( $\star$ ), shown in Fig. 21, is taken into the cell site, and eventually absorption (A) of the matrix occurs. The tonofibrils are arranged along the cell axis. $\times 13,900$. 
Plate IV

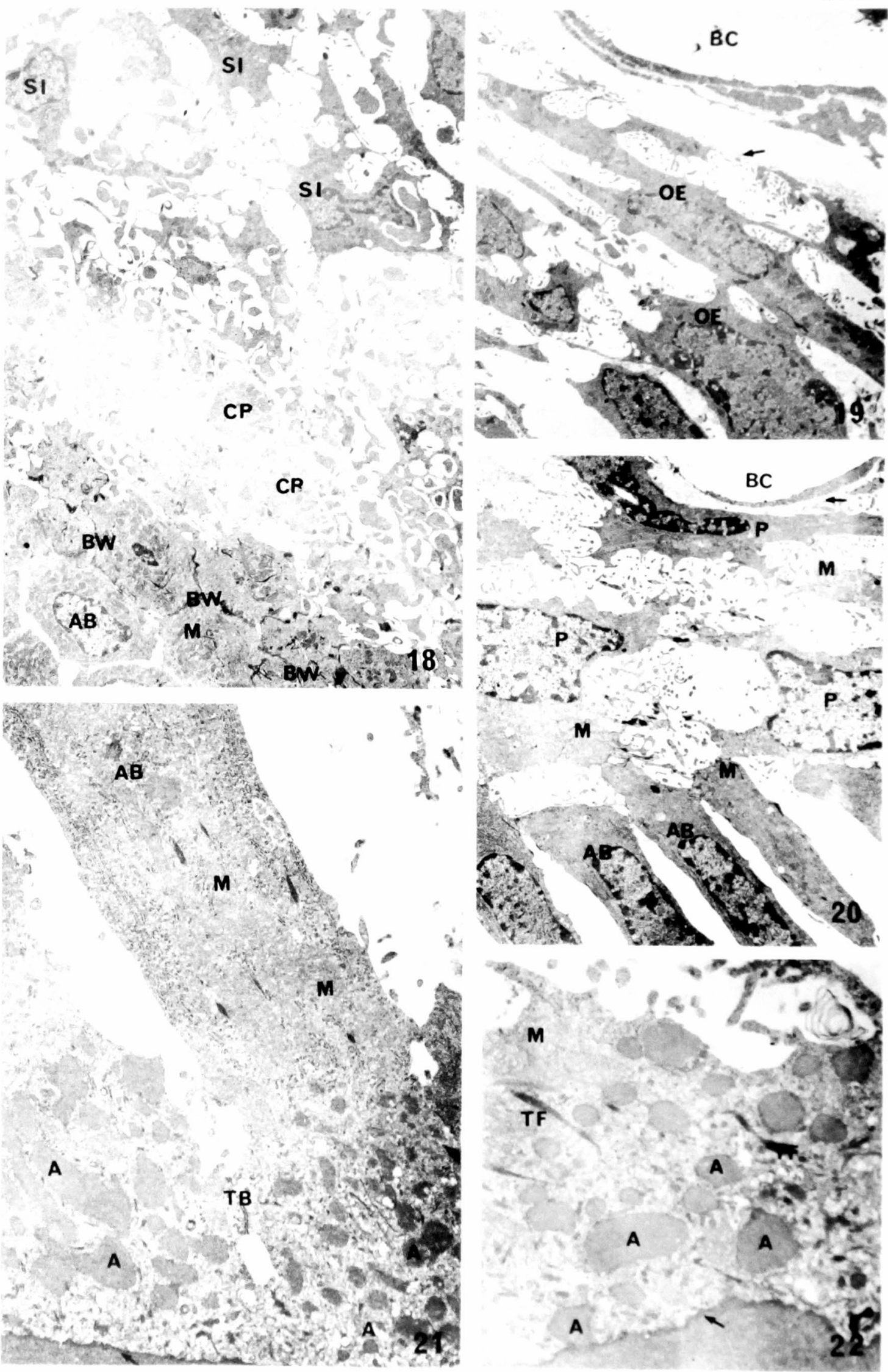




\section{Plate V}

Fig. 23. Ruffle-ended ameloblasts (RA) and papillary cells of the late maturation zone. The RA have wide intercellular spaces, which continue directly to those of the papillary cells, with many microvilli. Infoldings (IF) are found in the distal end of the RA. The mitochondria are located both basally and distally. $(\leftarrow)$ the liner. $\times 2,870$.

Fig. 24. Smooth-ended ameloblasts (SA) of the late maturation zone. Intercellular space is not found and infoldings are not present in the distal end. Basal and discal mitochondria are found. $(\leftarrow)$ the liner. $\times 4,130$.

Fig. 25. Distal end of the RA. Variously sized dense granules (DG) are seen around the infoldings (IF). $(\leftarrow)$ the liner. $\times 13,000$.

Fig. 26. Distal end of the SA. The infoldings disappear and the mitochondria are concentrated up to the distal end at least. $(\leftarrow)$ the liner. $\times 12,000$.

Fig. 27. Ameloblast and papillary cells of the early regression zone. $\times 5,000$.

Fig. 28. Corresponding cells of the late regression zones. $\times 5,000$.

Comparison between these two figures show that the intercellular spaces of the ameloblasts become narrower and the tonofibrils make up a network. The desmosomes between the papillary cells increase in number and width. 


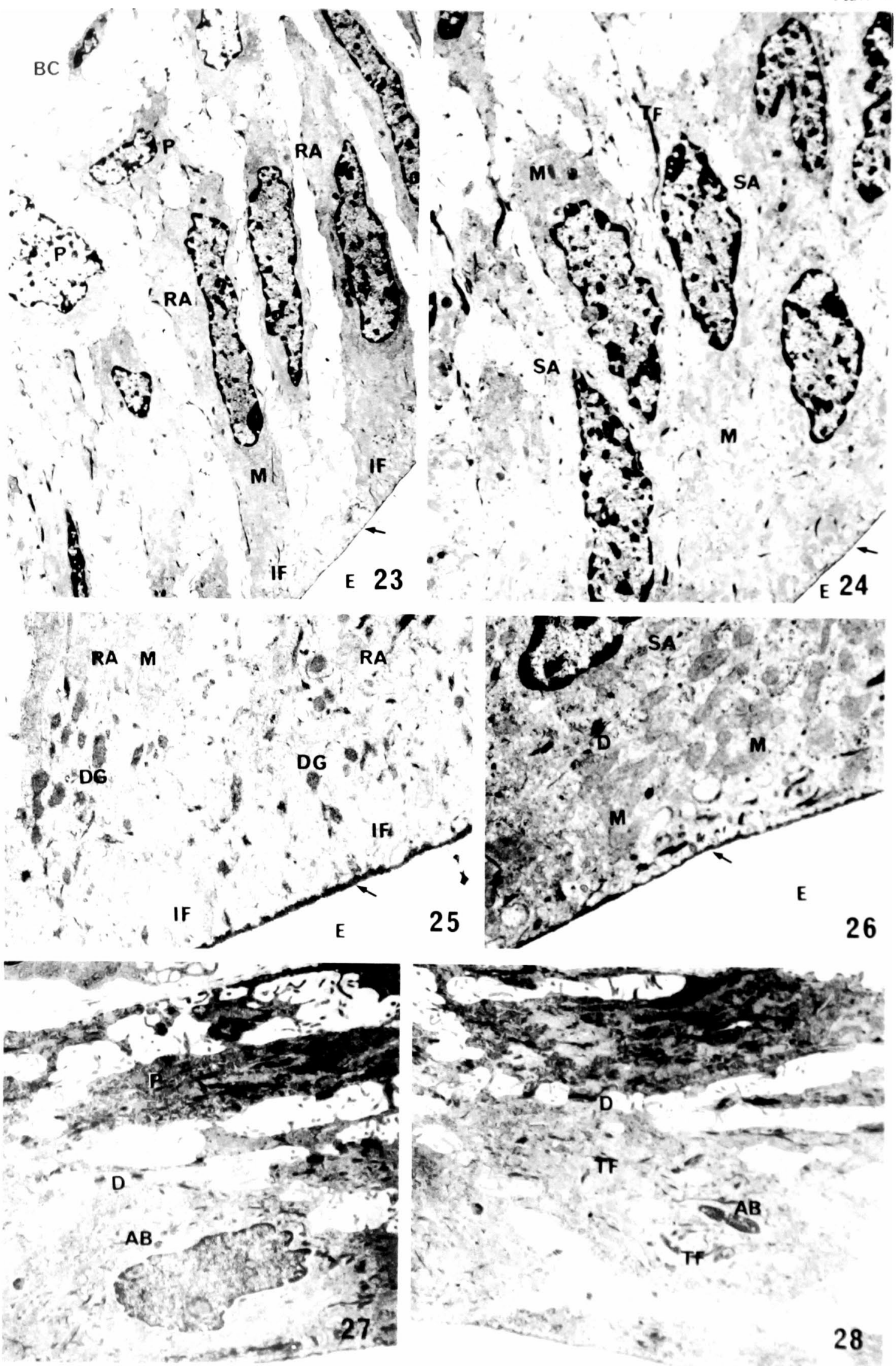

